\title{
A STUDY OF KARTAGENER'S SYNDROME PATIENTS AMONG 6 SITUS INVERSUS TOTALIS PATIENTS EVALUATED OVER 1 YEAR PERIOD AT ESIC- MC-PGIMSR
}

\author{
J. C. Panchasheelan ${ }^{1}$, Kavya K², Sai Shruthi' ${ }^{3}$, Jeetendra Kumar J. M4, Sathyanarayana N 5
}

\section{HOW TO CITE THIS ARTICLE:}

J. C. Panchasheelan, Kavya K, Sai Shruthi, Jeetendra Kumar J. M, Sathyanarayana N. "A Study of Kartagener's Syndrome Patients Among 6 Situs Inversus Totalis Patients Evaluated over 1 year period at ESIC-MC-PGIMSR". Journal of Evolution of Medical and Dental Sciences 2014; Vol. 3, Issue 24, June 16; Page: 6694-6697, DOI: $10.14260 /$ jemds/2014/2806

\begin{abstract}
Situs inversus totalis (SIT) entails a mirror-image reversal of all the asymmetrical structures of the body. Many people with situs inversus are unaware of the condition. Kartagener's syndrome (KS) is a subset of a larger group of ciliary motility disorders called primary ciliary dyskinesias, comprising a triad of situs inversus, bronchiectasis and sinusitis. We found 6 cases of Situs inversus totalis (SIT) over one year period among admitted patients in our department at ESIC MC PGIMSR and on evaluation 2patients were found to have Kartagener's syndrome (KS). As the syndrome is rare we hereby report the manifestations and findings in detail.
\end{abstract}

KEYWORDS: Situs inversus, primary ciliary dyskinesia, Kartagener's syndrome, sinusitis, bronchiectasis.

INTRODUCTION: Situs inversus total (SIT) entails a mirror-image reversal of all the asymmetrical structures of the body and it is estimated to occur in 1/8000-1/25 000 newborns.[1] Many people with situs inversus are not aware of their unusual anatomy until they come for medical evaluation for other conditions. Kartagener's syndrome (KS) comes under larger group of ciliary motility disorders called primary ciliary dyskinesias (PCDs). The estimated prevalence of PCD also known as "immotile ciliary syndrome", is about 1 in 30,000 though it may range from 1 in 12,500 to 1 in 50,000.[2] Kartagener's syndrome (KS) is a genetic condition with an autosomal recessive inheritance, comprising a triad of situs inversus, bronchiectasis and sinusitis. Siewart first described this condition in 1904, then it was Kartagener who recognized the etiological correlation between various elements of the triad and reported four cases in 1933.[3] We found 6 cases of Situs inversus totalis (SIT) over one year period among admitted patients in our department and on evaluation 2 patients were found to have Kartagener's syndrome (KS). As the syndrome is rare we hereby report the manifestations and findings in detail.

A high index of suspicion is needed to detect Kartagener's syndrome (KS) early so that treatment options, if feasible can be considered for infertility in these patients. It seems likely that early diagnosis is important for the preservation of pulmonary function, quality of life and life expectancy in these patients.[4] However, further large prospective studies are needed for confirmation.

CASE DESCRIPTION: Over one year period, between April 2013 and March 2014, among patients admitted under our department, 6 patients (4 males and 2 females) were diagnosed with (SIT) Situs Inversus Totalis. Out of these 6 patients, 2 male patients were diagnosed to have Kartagener's Syndrome (KS). The diagnosis of KS was based on the demonstration of the classic triad of the 
syndrome, consisting of bronchiectasis, paranasal sinusitis and SIT. These patients underwent a similar diagnostic study that included sputum culture for aerobic microorganisms, including Mycobacterium tuberculosis, electrocardiogram, complete spirometry, abdominal ultrasound, computed tomography (CT) with contrast as well as high resolution CT (HRCT) and CT of paranasal sinuses (PNS). By means of CT, the severity of the bronchiectasis and sinusitis was evaluated. A semen analysis was done with consent of patients.

Among the 4 patients with only situs inversus totalis (SIT), ( 1 male and 1 female) 2 patients were admitted for dengue fever and thrombocytopenia; SIT was diagnosed incidentally. 1 male patient was admitted with viral hepatitis and SIT was diagnosed during abdominal USG and further evaluation. 1 female patient was admitted for iron deficiency anemia and bilateral CSOM which was operated later.

Case 1: A 44years-old man presented to the outpatient department with complaints of recurrent episodes of common cold, cough with sputum for past 15 years, shortness of breath on exertion for last 10 years. On eliciting detailed history we found he is not having children despite being married for last 16 years. The patient frequently developed cough, cold, nasal blockade and ear discharge during childhood. He was born to parents out of non consanguineous marriage, had 2 female siblings who are keeping normal health. On examination, the vital parameters were within normal limits. Physical examination revealed grade 3 clubbing in fingers and apex beat on the right side in fifth intercostal space. On auscultation, bilateral basal crackles were audible and heart sounds best heard on the right side of the chest. Electrocardiogram showed evidence of dextrocardia. Chest X-ray Postero-anterior (PA) revealed cardiac apex and aortic arch on the right side, suggesting dextrocardia. Radiograph of sinuses showed mucosal thickening in maxillary sinuses. An ultrasound of the abdomen revealed a normal liver, gall bladder on the left side and a normal spleen on the right side. Contrast-enhanced computed tomography (CECT) chest revealed dextrocardia and nodular opacities in both lower lobes, suggestive of bronchiectasis. A semen analysis revealed immotile sperms.

CASE 2: A 40years-old male patient, non-smoker and born to non-consanguineous parents presented with recurrent productive cough and headache since last 20 years with episodic fever and worsening of symptoms. He had been previously treated with antibiotics, antihistamines, bronchodilators, inhaled and oral corticosteroids. He also had similar complaints on and off during childhood. On examination, he was febrile and had bilateral coarse crackles. His heart sounds were heard best on right side of the chest. Chest X-ray showed cystic bronchiectactic changes in the lower and mid zones with dextrocardia. Ultrasound of the abdomen showed spleen on the right side of the abdomen, while liver on the left side. Axial CT-chest showed dextrocardia. Axial CT-abdomen showed situs inversus with liver, IVC on the left and spleen and aorta on the right. HRCT- chest showed dilated and thickened medium-sized airways. Axial CT-paranasal sinuses showed mucosal thickening and sinus opacification. Semen analysis showed oligospermia with immotile live sperms.

DISCUSSION: Siewert first described combination of situs inversus, chronic sinusitis, bronchiectasis in 1904. In 1933 Manes Kartagener first recognized the clinical triad as a distinct congenital syndrome in detail, so it bears his name. Kartagener's syndrome (KS) is inherited via an autosomal 
recessive pattern. Male patients with this syndrome are almost invariably infertile because of immotile spermatozoa. The non-motility is due to variety of ultra structural defects in respiratory cilia and sperm tail.[4]

Sinusitis, bronchiectasis, situs inversus and male infertility in Kartagener's syndrome (KS) are attributed to abnormal ciliary motility. Cilia may be immotile or may show uncoordinated and inefficient movement patterns. In 1975 Camner and coworkers first suggested ciliary dyskinesia as the cause of Kartagener's syndrome (KS).[5] The diagnostic criteria recommended for this syndrome are history of recurrent chronic bronchial infection from early childhood, combined with one or more of: (a) situs inversus or dextrocardia in a patient or a sibling (b) live but immotile spermatozoa (c) near absence of tracheobronchial clearance. ${ }^{[6,7]}$

Treatments include antibiotics, intravenous or oral, intermittent or continuous and are used to treat upper and lower airway infections. Obstructive lung disease/bronchiectasis should be treated with inhaled bronchodilators, mucolytics, and chest physiotherapy.

CONCLUSION: The correct diagnosis of this rare congenital autosomal recessive disorder in early life is important in the overall prognosis of the syndrome, as many of the complications can be prevented if timely management is instituted. Late diagnosis with established bronchiectasis worsens the overall the prognosis, even with the best of treatment modalities.

\section{REFERENCES:}

1. Tabry IF, Calabrese J, Zammar H, Abou-Kasem K, Akeilan H, Gharbieh N, Zinati H, Noureddine W, el-Hout A, Tayah M, Khalidy L, Yaghi M. Case report: off-pump total myocardial revascularization for dextrocardia and situs inversus. Heart Surg Forum. 2001; 4: 251-253.

2. Seaton D. Bronchiectasis. In: Seaton A, Seaton D, Leitch AG, editors. Crofton and Douglas's respiratory diseases. $5^{\text {th }}$ ed. Oxford: Blackwell Science; 2004. p. 794-828.

3. Dixit R, Dixit K, Jindal S, Shah KV. An unusual presentation of immotile-cilia syndrome with azoospermia: Case report and literature review. Lung India 2009; 26: 142-5.

4. Barbato A, Frischer T, Kuehni CE, Snijders D, Azevedo I, Baktai G, et al. Primary ciliary dyskinesia: A consensus statement on diagnostic and treatment approaches in children. Eur Respir J 2009; 34: 1264-76.

5. Samuel I. Kartagener's syndrome with normal spermatozoa. JAMA 1987; 258: 1329-30.

6. Camner P, Mossberg B, Afzelius BA. Evidence of congenitally non-functioning cilia in the tracheobronchial tract in two subjects. Am Rev Respir Dis 1975; 112:807-9.

7. Bent J III. Kartagener syndrome. e medicine 2009; 69:39-41. 


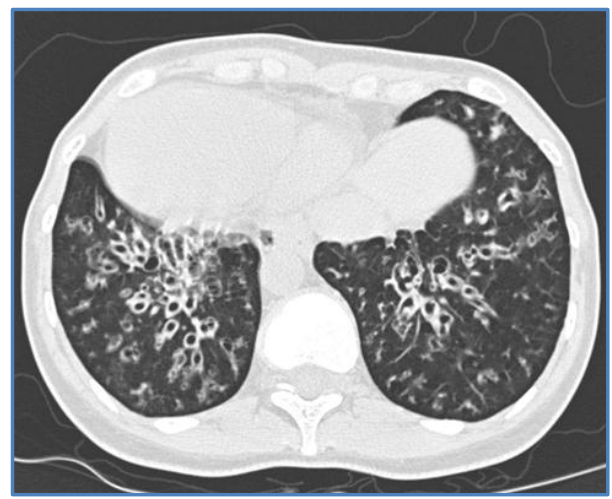

\section{Fig. 1: karatageners syndrome with bronchiectasis}

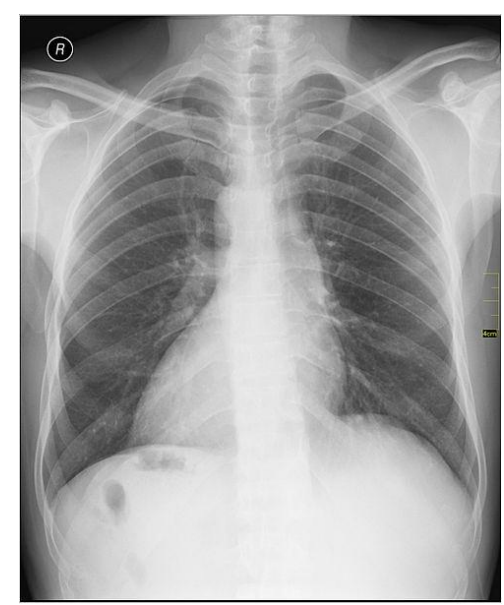

\section{Fig. 2: Chest X-ray showing dextrocardia}

\section{AUTHORS:}

1. J. C. Panchasheelan

2. Kavya K.

3. Sai Shruthi

4. Jeetendra Kumar J. M.

5. Sathyanarayana N.

\section{PARTICULARS OF CONTRIBUTORS:}

1. Assistant Professor, Department of General Medicine, ESIC-MC- PGIMSR.

2. Post Graduate, Department of General Medicine, ESIC-MC- PGIMSR.

3. Post Graduate, Department of General Medicine, ESIC-MC- PGIMSR.

4. Professor and HOD, Department of General Medicine, ESIC-MC- PGIMSR.
5. Professor, Department of General Medicine, ESIC-MC- PGIMSR.

\section{NAME ADDRESS EMAIL ID OF THE CORRESPONDING AUTHOR:}

Dr. J. C. Panchasheelan, Assistant Professor, Department of General Medicine, ESIC-MC-PGIMSR, Bangalore - 560010.

E-mail: dr_panchasheel@yahoo.com

Date of Submission: 19/05/2014. Date of Peer Review: 20/05/2014. Date of Acceptance: 02/06/2014. Date of Publishing: 13/06/2014. 